\title{
Multi-level Analysis and Information Extraction Considerations for Validating 4D Models of Human Function
}

\author{
Kostas Marias $^{1}$, Dimitra D. Dionysiou ${ }^{2}$, Georgios S. Stamatakos ${ }^{2}$, \\ Fotini Zacharopoulou ${ }^{3}$, Eleni Georgiadi ${ }^{1,2}$, Thanasis Margaritis ${ }^{4}$, Thomas G. Maris ${ }^{3}$, \\ and Ioannis G. Tollis ${ }^{1}$ \\ ${ }^{1}$ Biomedical Informatics Laboratory, ICS-FORTH, Vassilika Vouton, P.O. Box 1385, \\ 71110 Heraklion, Crete, Greece \\ ${ }^{2}$ In Silico Oncology Group \\ Laboratory of Microwaves and Fiber Optics Institute of Communication and Computer \\ Systems School of Electrical and Computer Engineering National Technical University of \\ Athens Iroon Polytechniou 9, GR-157 80 Zografos, Greece \\ ${ }^{3}$ Medical Physics Department, University of Crete, Faculty of Medicine, Heraklion, Greece \\ ${ }^{4}$ Institute of Molecular Biology and Biotechnology, IMBB -FORTH, Vassilika Vouton, \\ P.O. Box 1385, 71110 Heraklion, Crete, Greece \\ kmarias@ics.forth.gr
}

\begin{abstract}
Recent research trends focus on how multiscale biomedical information can be modeled and transformed into knowledge, in order to lead to a less interfering but also more individualized diagnosis and therapy. In order to assess the clinical importance of models of human pathology (e.g. cancer), it is necessary to validate them with prior and post treatment clinical data which in turn requires the determination of the tumor size and shape with high resolution, accuracy and precision, as well as structural and physiological information. This paper discusses some of the most important image analysis challenges in order to define an optimal method for extracting more accurate and precise anatomical and functional information related to the underlying pathology, which can be used for initializing and validating models of pathophysiology as well as simulations/predictions of the response to therapeutical regimes.
\end{abstract}

Keywords: Virtual Physiological Human, biomedical data analysis, modeling.

\section{Introduction}

Modern scientific advances promise more efficient ways to optimally extract, analyze and model biomedical information in order to model pathophysiology. However, the integration of models from different levels remains an open issue. This paper addresses an important facet of this problem; the challenge of efficient and accurate extraction of biomedical information from different levels (from molecular to tissue/organ) in order to shed light in the computation of 4D maps of pathophysiological properties of tumors for model development and validation. 
Multi-level data analysis and information extraction are necessary steps for developing and validating mathematical models of human physiology and pathology.

Despite the recent advances in medical, molecular/genetic imaging, the robust extraction of physiological parameters remains an open issue since computing them from measurements (e.g. pixel values), isn't a trivial task. Although, pathophysiological measurements are essential for modeling human processes, the physics of imaging modalities (e.g. MRI) does not absolutely generalize; it is the physics of MRI electromagnetism or the ionizing radiation in CT that produces a representation of anatomy and physiology for interpretation. Also, the mechanism by which the image is formed inherently emphasizes certain aspects and provides less sensitivity to others.

In addition, traditional diagnostic imaging, no matter how sophisticated, is only a representation of the underlying anatomical and (patho) physiological characteristics of disease. Even the most sophisticated MRI and CT techniques can only achieve an order of magnitude below millimeter spatial resolution [1,2]. Therefore the information captured in the image is only an average of what is contained within the sampling range of the instrument. Given the local complexity and "multi-scale" nature of human physiology, any attempt to use imaging to capture detailed physiological processes will understandably generalize the underlying phenomena. Thus, care must be taken when interpreting any kind of physiological parameter from imaging. This is particularly true in contrast-enhanced (CE) MRI analysis where the choice of a different analysis models may lead to a completely different result regarding the true spatial extent of a cancer.

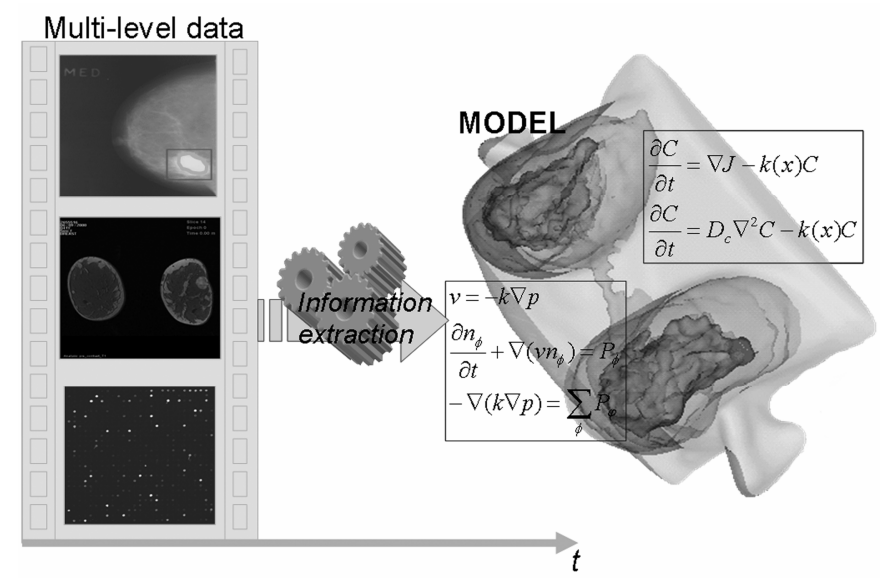

Fig. 1. Extraction of temporal pathophysiological information is essential for developing and validating multi-level models

The above considerations are very important for the development of computational frameworks for multi-level (from molecular/genetic to tissue/organ) modeling and simulation of human pathophysiology. Multi-level measurements (e.g. molecular/genetic, diagnostic imaging, etc.), should be properly interpreted and 
combined in order to be used in simulation models of human function. Additionally, this process needs to be repeated in several time instances in order to assess the validity of each model temporally. This concept is schematically illustrated in Fig. 1 where information extraction of temporal multi-level data is driven into the corresponding multi-level model of breast cancer. To reach this goal, it is essential to be able to optimally extract robust temporal pathophysiological information from multi-level measurements (e.g. microarrays, CE MRI, etc.).

Medical Imaging has focused in providing anatomical information, mainly imaging human bones, dense tissue and arteries. Recent advances especially in PET and functional MRI allowed the study of various pathological processes via radio-labelled tracers (PET) or pharmaco-kinetic models in contrast enhanced MRI. The whole field of molecular medicine and molecular imaging is opening up new possibilities for targeted assessment of disease and disease mechanisms. Also, microarray imaging has created exiting possibilities for measuring gene differential expression and defining new disease biomarkers. Concluding, from the imaging standpoint it is essential to consider the following points relevant to human pathophysiology modeling:

a) There is a need for a holistic understanding of pathophysiology and this clearly implies a multidisciplinary approach. To this end, molecular and genetic imaging offer unique opportunities to better understand pathophysiology in smaller scales and built multilevel models. As an example, one can think of the crucial role of imaging the genetic profile changes in cancer modeling as well as global changes in tumour size, density, etc. by analysing temporal medical imaging data.

b) It is necessary to pre-process biomedical data at all possible scales (e.g. medical images, microarray scans) in order to extract all the information that is needed for a given model. This way, multiscale information extraction aims to 'individualise' a given model.

The next section focuses on specific issues regarding the previous points.

\section{Problems Related to Multi-level Physiological Information Extraction}

\subsection{Limitations in Extracting Pathophysiological Information from Medical Images}

It is always essential to use of some kind of process (e.g. segmentation) to identify important structures and features in medical images (e.g. tumours can be segmented using a pharmacokinetic model of gadolinium uptake with contrast-enhanced MRI, while microarray spots can be segmented by combining the two different information channels i.e. $\mathrm{Cy} 3$ and $\mathrm{Cy} 5$ [7]). As mentioned before, the intrinsic limitations of imaging technologies often are responsible for problems related to the assessment of subtle pathophysiological changes in micro-structure. The main problem is that in order to characterize the function of an organ it is essential to acquire multi-modality imaging data, since each imaging modality produces a representation that has different parametric properties. For example, the active volume of a tumor detected with X-ray mammography is less accurate than that using with MRI. 
In cancer imaging, perfusion is a complex process that encompasses the delivery of nutrients (primarily oxygen and glucose), their diffusion and convection into the tumor parenchyma, and the removal of waste products. Therefore, any perfusion information is crucial for characterizing the tumor microenvironment. The perfusion of tumors can be interrogated with time-dependent delivery of exogenous contrast, using contrast agents. Dynamic contrast-enhanced magnetic resonance imaging (DCE-MRI) is in most cases applied by the clinicians in order to decide upon the state of the tumor. This is performed after the administration of intravenous contrast medium in order to noninvasively assess tumor vascular characteristics. Kinetic parameters can be then correlated with immunohistochemical surrogates of tumor angiogenesis, including microvessel density, and with pathologic tumor grade. DCEMRI is being applied to monitor the clinical effectiveness of a variety of treatments, including antiangiogenic drugs. Kinetic parameter changes following treatment have been correlated with histopathological outcome and patient survival [8].

However, when no DCE-MRI image data could be acquired a standard CE-MRI should be used to trace the tumor. The success of this technique depends on its ability to demonstrate quantitative differences of contrast medium behavior in a variety of tissues. In particular, malignant tumors exhibit an increased vascularity, since they begin to grow their own blood supply network. For this reason when the contrast agent is distributed, malignant masses enhance faster. This led to the development of models of contrast uptake as is illustrated in Figure 2.

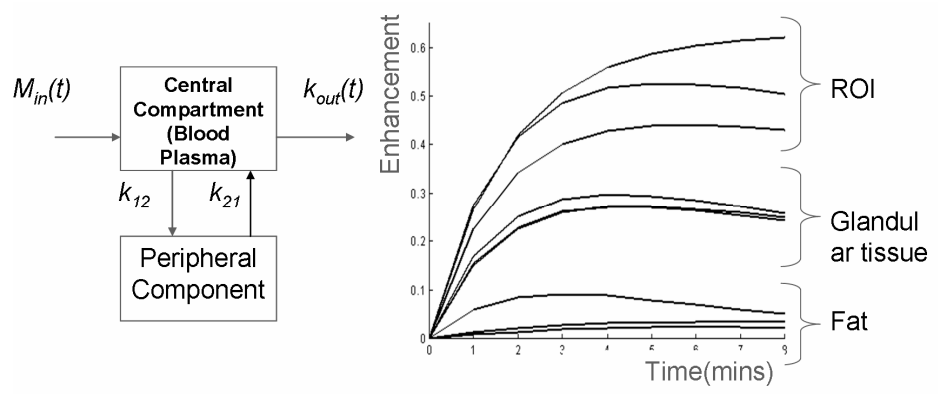

Fig. 2. A two-compartment pharmacokinetic model with typical contrast curves for fat, parenchymal (glandular) tissue and enhancing regions of interest. $M_{i n}$ is the mass of contrast injected into the blood stream with respect to time. $k_{12}$ and $k_{21}$ are inter-compartment exchange rates and $k_{\text {out }}$ is the leaving contrast rate.

This technique can be misleading on several conditions. It is common knowledge that the contrast media perfuse over time in tissue. There is no evidence that after a specific time interval the contrast agent perfuse by tumor tissue exclusively. In addition, it has been observed that tumor tissue 'liquifies' after treatment due to destruction of the protein structure. Quantitative MRI (qMRI) techniques can actually quantify this alteration of the tissue structure by measuring its physical parameters however this technique isn't routinely used in clinical practice.

In conclusion, there are several limitations in extracting actual pathophysiological information from medical images. Therefore, in order to build robust models it is 
essential to carefully chose the imaging modalities and use as much complementary information as possible. However, the information extraction process is difficult to generalize and specific tools should be combined when different modalities are used (e.g. the two-compartment pharmacokinetic model for segmentation shown in Fig. 2). In the case of cancer modeling, several scientists have suggested the inclusion of qMRI techniques in the clinical protocols associated with the validation of insilico models since the alteration of the tissue structure, detected by MRI, could shed light in the accurate delineation of the tumor especially in areas where DCE technique is unreliable (e.g. the periphery of the tumor). As an example of possible multimodal information fusion, the combination of DCE-MRI and parametric qMRI could provide more accurate and precise tumor information, which would serve as a basis for validating insilico models within a higher level confidence.

\subsection{Geometrical Normalization}

It is necessary to ensure that a point correspondence is computed between different images. This is a typical problem in the case of breast imaging owing to the differences in breast shape/compression but also in newer applications such as molecular imaging and microarray imaging. Non-rigid alignment or registration is required to compensate for such differences. This problem does not only pertain to the multi-modal scenaria, as a temporal acquisition of the same modality will still likely involve registration in order to facilitate comparison. Several registration frameworks have been proposed (see [3-5] for selected publications in the field), traditionally for medical imaging applications but more recently also for correcting time dependent geometries in 2D molecular optical imaging studies [6].

\subsection{Intensity Normalization}

As stated above each biomedical measurement in essence disguises the true physiological property due to the image formation process. The non-linearities introduced by varying imaging conditions may alter significantly the image-intensity profile and reduce the efficiency of generic analysis algorithms. An interesting example is the model of Highnam and Brady [9] for mammogram image normalisation that eliminates variations related to imaging conditions (e.g. tube voltage, time of exposure, etc). Highnam and Brady's method estimates - in millimetres - the amount of interesting tissue in each pixel column. This effectively provides objective quantitative information about the breast anatomy. If, for example, the separation between the Lucite plates is $6.5 \mathrm{~cm}$, the amount of interesting tissue at a location $(\mathrm{x}, \mathrm{y})$ might be $4.75 \mathrm{~cm}$, implying $1.75 \mathrm{~cm}$ of fat. This way, the algorithm estimates, and then eliminates the effects of, the particular parameters that were used to form the mammographic image providing true anatomical information.

This problem also exists in microarray imaging technologies where several nonlinearities in the experimental process render the measured expression values prone to variability and often, to poor reproducibility. To achieve normalization, one has to adjust the sensitivity of detection (photomultiplier voltage with fluorescence or exposure time with radioactivity) so that the measurements occupy the same dynamic range in the detector and exploit the fact that the gene expression values (e.g. from the 
Cy3 and Cy5 matrices), should ideally follow a linear trend [10]. The later can be performed to all the genes or to a 'ground truth' subset that is known a priori to be the same in both channels (Cy3 and Cy5).

\subsection{Visualisation}

This is a fundamental aspect of biomedical data information fusion that is typically less well addressed in the literature, but which can dramatically increase the clinical utility of a solution if implemented intelligently. The effectiveness of visualisation depends very strongly on how clearly different indicators can be extracted from data and therefore segmentation is of utmost importance. A great deal of effort has been made in this research to produce visualizations of the results of temporal and multimodal image fusion that optimizes the presentation of available clinical information (an example is illustrated in Figure 3).

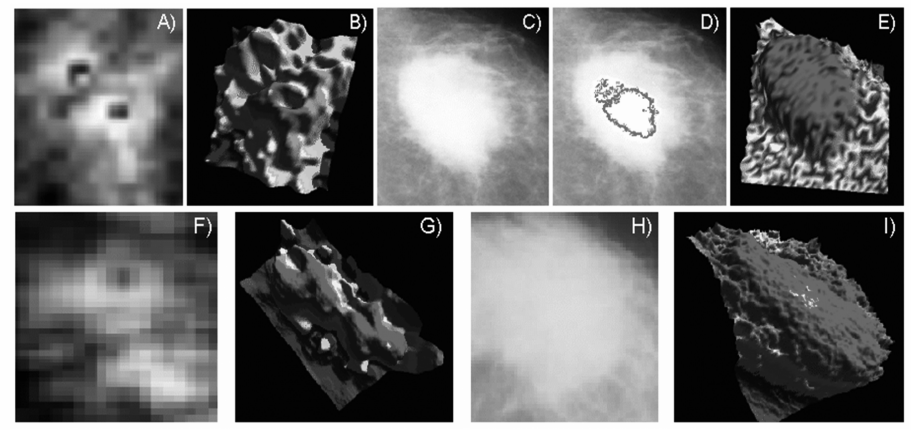

Fig. 3. A-D and F-I: Multimodal (MRI and X-ray) 3D visualization of 2D medical images better highlights the necrotic centers of each tumour

\section{Conclusions}

In order to approach the vision of the Virtual Physiological Human, it will be essential to develop and validate individualized, multi-level models taking into consideration pathophysiological information at all scales. As discussed in this paper, the extraction of useful anatomical and physiological information from biomedical measurements isn't a trivial task due to the complex physical interactions involved in each acquisition as well as several systematic and random errors involved in the process. In many cases, the problems that arise in different scales are common (e.g. geometrical inconsistencies over time) and is therefore important to develop generic tools for multi-scale temporal analysis in order to robustly extract and visualize pathophysiological information over time. Such information is crucial for initializing (i.e. in the case of in silico models of cancer, 3D voxels should be classified as 'proliferating', 'necrotic', etc.), inspiring and validating 4D models of human function. 


\section{References}

1. Behrenbruch, C.P., Marias, K., Armitage, P.A., Yam, M., Moore, N.R., English, R.E., Clarke, P.J., Leong, F.J., Sir Brady, J.M.: Fusion of contrast-enhanced breast MR and mammographic imaging data. British Journal of Radiology 77, 201-208 (2004)

2. Behrenbruch, C.P., Marias, K., Armitage, P.A., Yam, M., Moore, N., English, R.E., Clarke, P.J., Brady, M.: Fusion of Contrast-Enhanced Breast MR and Mammographic Imaging Data. In: Medical Image Analysis (MedIA), vol. 7(3), pp. 311-340. Elsevier, Amsterdam (2003)

3. Guimond, A., Roche, A., Ayache, N., Meunier, J.: Multimodal Brain Warping Using the Demons Algorithm and Adaptative Intensity Corrections. IEEE Transaction on Medical Imaging 20(1), 58-69 (2001)

4. McInerney, T., Terzopolous, D.: Deformable models in medical image analysis: a survey. In: Medical Image Analysis, pp. 91-108. Oxford University Press, New York (1996)

5. Penney, G.P., Weese, J., Little, J.A., Hill, D.L.G., Hawkes, D.J.: A comparison of similarity measures for use in 2D-3D medical image registrtation. IEEE Transactions in Medical Imaging 17, 586-595 (1998)

6. Marias, K., Ripoll, J., Meyer, H., Ntziachristos, V., Orphanoudakis, S.: Image Analysis for Assessing Molecular Activity Changes in Time-Dependent Geometries. IEEE Transactions on Medical Imaging, Special issue on Molecular Imaging 24(7) (July 2005)

7. Margaritis, T., Marias, K., Kafetzopoulos, D.: Improved Microarray Spot Segmentation by Combining two Information Channels. In: proceedings of the 2006 IEEE Engineering in Medicine and Biology Society (EMBS) Annual International Conference, IEEE. 2006, New York, USA (2006)

8. Bhujwalla, Z.M., Artemov, D., Glockner, J.: Tumor angiogenesis, vascularization,and contrast-enhanced magnetic resonance imaging. Top Magn Reson Imaging 10, 92-103 (1999)

9. Highnam, R.P., Brady, J.M.: Mammographic Image Analysis. Kluwer Academic Publishers, Dordrecht (1999)

10. Chen, Y., Dougherty, E.R., Bittner, M.L.: Ratio-based decisions and the quantitative analysis of cDNA microarray images. J. Biomed. Optics 2, 364-374 (1997) 